\title{
Isolation, Identification, Molecular and Electronic Structure, Vibrational Spectroscopic Investigation, and Anti-HIV-1 Activity of Karanjin Using Density Functional Theory
}

\author{
Anoop kumar Pandey, ${ }^{1}$ Abhishek Kumar Bajpai, ${ }^{2}$ Ashok Kumar, ${ }^{3}$ Mahesh Pal, \\ Vikas Baboo, ${ }^{3}$ and Apoorva Dwivedi ${ }^{2}$ \\ ${ }^{1}$ Department of Physics, Government Danteshwari P.G.College, Dantewada 494449, India \\ ${ }^{2}$ Department of Physics, Government Kakatiya Post Graduate College, Jagdalpur, Bastar, Chhattisgarh 494001, India \\ ${ }^{3}$ Department of Chemistry, Lucknow University, Lucknow 226007, India \\ ${ }^{4}$ National Botanical Research Institute, Lucknow 226007, India \\ Correspondence should be addressed to Apoorva Dwivedi; apoorvahri@gmail.com
}

Received 17 January 2014; Revised 9 April 2014; Accepted 9 April 2014; Published 7 May 2014

Academic Editor: Hugo Verli

Copyright (C) 2014 Anoop kumar Pandey et al. This is an open access article distributed under the Creative Commons Attribution License, which permits unrestricted use, distribution, and reproduction in any medium, provided the original work is properly cited.

\begin{abstract}
"Karanjin" (3-methoxy furano-2,3,7,8-flavone) is an anti-HIV drug, and it is particularly effective in the treatment of gastric problems. The method of isolation of "Karanjin" followed the Principles of Green Chemistry (eco-friendly and effortless method). The optimized geometry of the "Karanjin" molecule has been determined by the method of density functional theory (DFT). Using this optimized structure, we have calculated the infrared wavenumbers and compared them with the experimental data. The calculated wavenumbers are in an excellent agreement with the experimental values. On the basis of fully optimized ground-state structure, TDDFT//B3LYP/LANL2DZ calculations have been used to determine the low-lying excited states of Karanjin. Based on these results, we have discussed the correlation between the vibrational modes and the crystalline structure of "Karanjin." A complete assignment is provided for the observed FTIR spectra. This is the first report of the isolation, molecular and electronic structure using vibrational spectroscopic investigation, density functional theory, and anti-HIV-1 activity of "Karanjin."
\end{abstract}

\section{Introduction}

Pongamia pinnata is a medium sized glabrous tree, found throughout Indian forests [1]. Different parts of this plant have been used as a source of traditional medicine. P. pinnata seeds contain oil which is mainly used in tanning industry for dressing of leather and to some extent it is used in soap industry. Oil is employed in scabies, herpes, and leucoderma, and sometimes as stomachic and cholagogue in dyspepsia and sluggish liver [2]. "Karanjin" is an active principle responsible for the curative effects of the oil in skin disease [1]. Seed extract inhibits growth of herpes simplex virus and also possesses hypoglycemic, antioxidative, antiulcerogenic, anti-inflammatory, and analgesic properties [3]. During the course of exploration of new compounds from $P$. pinnata seed oil, several workers [4-6] have identified some new compounds of its seed oil apart from "Karanjin." "Karanjin" possess pesticidal [7], insecticidal [8], and anti-inflammatory activity [9].

Considering the role of "Karanjin" in different areas, in the present communication, we have carried out isolation and identification of "Karanjin" by ecofriendly method and tested for its anti-HIV activity. The molecular structure of the wellknown natural product "Karanjin" has been studied using the density functional theory. The equilibrium geometry, harmonic vibrational frequencies, and HOMO-LUMO gap have been calculated by the density functional B3LYP method employing 6-311 G (d, p) as the basis set. The detailed interpretation of the vibrational spectra of Karanjin in terms of the normal mode analysis has been reported. The main objective of the present study is to investigate in detail the vibrational spectra of the important biological molecule (Karanjin) by DFT [10] method, which can presumably help 


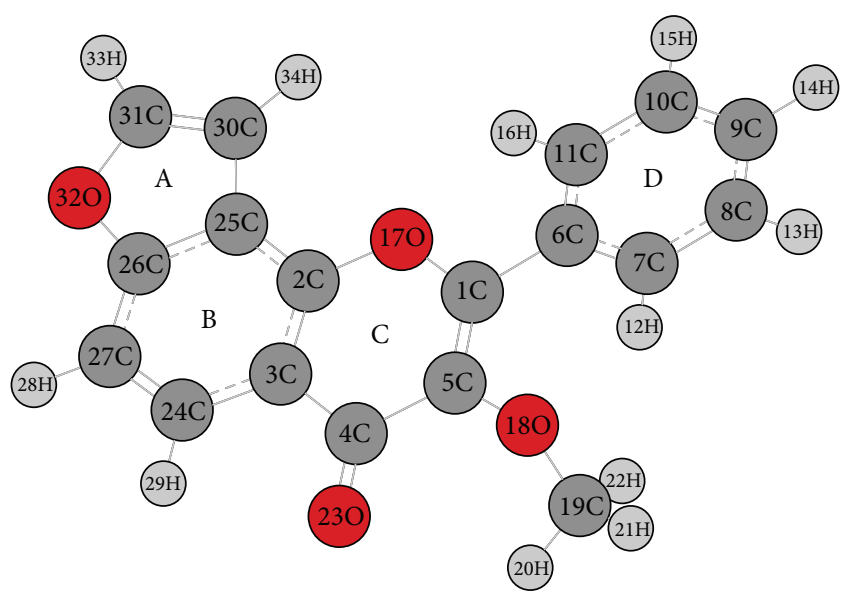

FIgURE 1: Model molecular structure of Karanjin.

in understanding its dynamical behavior. To the best of our knowledge, no detailed DFT calculations and anti-HIV activity have been performed on "Karanjin" so far in the literature.

\section{Experimental Methods (Structure and Spectra)}

The molecular structure of the title compound "Karanjin" is made by molecular modeling. The model molecular structure of the compound is given in Figure 1. Fourier transform infrared spectrum was recorded with FTIR Perkin Elmer spectrometer in $\mathrm{KBr}$ dispersion in the range of 500 to $4000 \mathrm{~cm}^{-1}$ for the title molecule. The comparison of the calculated and experimental FTIR and UV visible spectra of "Karanjin" is given in Figures 2 and 3, respectively.

\section{Computational Methods}

The initial geometry was generated from the standard geometrical parameters and was minimized without any constraint in the potential energy surface. The gradient corrected density functional theory (DFT) with the three-parameter hybrid functional (B3) [11] for the exchange part and the LeeYang-Parr (LYP) correlation function [12] has been employed for the computation of molecular structure, vibrational frequencies, HOMO-LUMO, and energies of the optimized structures, using Gaussian 09 [13]. The calculated vibrational frequencies have also been scaled by a factor of 0.963 [14]. By combining the results of the GaussView program [15] with symmetry considerations, vibrational frequency assignments were made with a high degree of accuracy. We used this approach for the prediction of IR frequencies of title compound and found it to be very straightforward. Density functional theory calculations are reported to provide excellent vibrational frequencies of organic compound if the calculated frequencies are scaled to compensate for the approximate treatment of electron correlation, for basis set deficiencies and for anharmonicity. A number of studies have been carried out regarding calculations of vibrational spectra

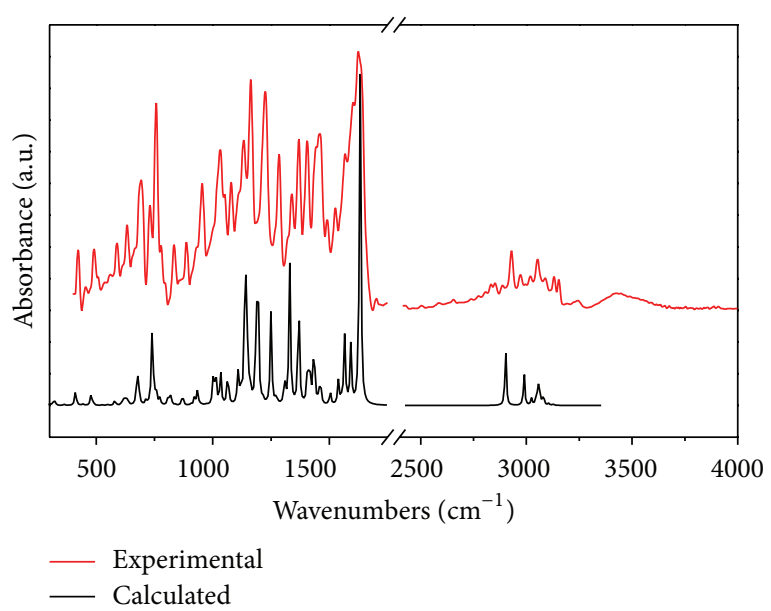

FIGURE 2: Comparison of calculated and experimental FTIR spectra of Karanjin.

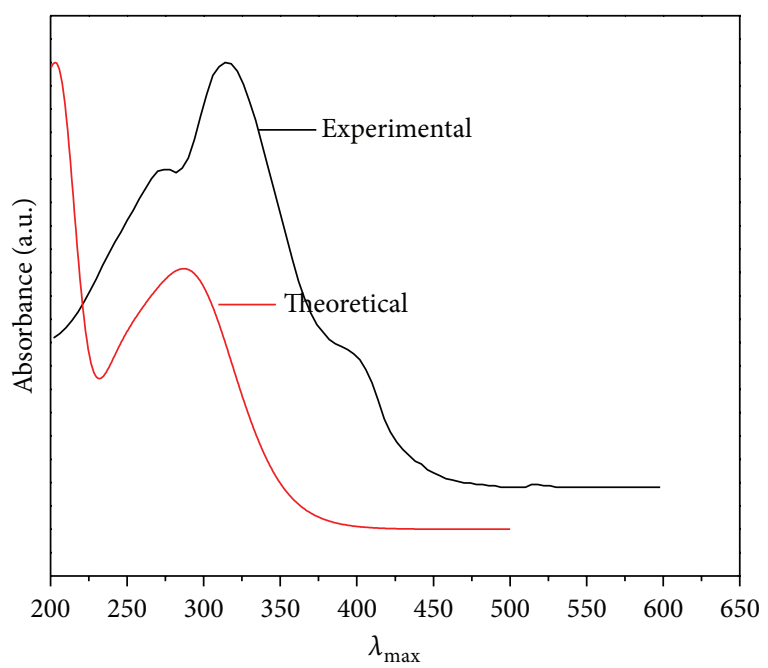

FIGURE 3: Comparison of calculated and experimental UV visible spectra of Karanjin.

by using B3LYP methods with 6-311 G (d, p) basis set. The scaling factor was applied successfully for B3LYP method and was found to be easily transferable in a number of molecules. Thus, vibrational frequencies calculated by using the B3LYP functional with 6-311G $(\mathrm{d}, \mathrm{p})$ as basis set can be utilized to eliminate the uncertainties in the fundamental assignment in the IR spectra.

\section{Results and Discussion}

4.1. Eco-Friendly Method. Here it needs to be highlighted that so far "Karanjin" has been isolated through column chromatography (silica gel, 100-200 mesh) or by preparative HPLC [16-18], but in our study the method was ecofriendly and effortless and followed the Principles of Green Chemistry. Implementing these Green Chemical Principles requires a certain investment, since the current, very inexpensive chemical processes must be redesigned. A typical chemical process generates products and wastes from raw 
materials such as substrates, solvents, and reagents. If most of the reagents and the solvent can be recycled, the mass flow looks quite different.

4.2. Isolation of "Karanjin". The shade dried Karanja seeds (Pongamia pinnata) of $3.5 \mathrm{~kg}$ were extracted with methanol $(\mathrm{MeOH})(4.5$ lit) at room temperature. The combined $\mathrm{MeOH}$ extract was concentrated under reduced pressure at $40^{\circ} \mathrm{C}$ to a dark viscous mass. It was concentrated to dryness and kept at $4^{\circ} \mathrm{C}$ for $24 \mathrm{hr}$; after adding ethanol, shake it properly and keep on for settling down of crystals for few hours. Colorless crystals (11.2 g) were obtained from crystallization with EtOH isolated with TLC in 98:2 chloroform and $\mathrm{MeOH}$ and a single spot was obtained.

4.3. Identification of "Karanjin". Isolated compound identified as "Karanjin" (3-methoxy furano-(20,30:7,8)-flavone) by direct comparison of co-TLC and melting point of $162^{\circ} \mathrm{C}$ with that of authentic sample obtained from Sigma-Aldrich and was also confirmed by the ${ }^{1} \mathrm{H}$ NMR and ${ }^{13} \mathrm{C}$ NMR reported in the literature [19].

4.4. Cytotoxicity and Anti-HIV-1 Activity of Compound. Compound "Karanjin" was tested for cytotoxicity against C8166 cells (CC50), and anti-HIV-1 activity was evaluated by the inhibition assay for the cytopathic effects of HIV-1( $\left.\mathrm{EC}_{50}\right)$ $<$ using AZT as a positive control; the compound exerted moderate cytotoxic activity against $\mathrm{C} 8166$ cells with $\mathrm{CC}_{50}>$ $693.15 \mu \mathrm{M}$ and showed anti-HIV-1 activity with $\mathrm{EC}_{50}=$ $49.43 \mu \mathrm{M}$ and selectivity index $\left(\mathrm{CC}_{50} / \mathrm{EC}_{50}\right)$ more than 14.02 . Cytotoxicity and anti-HIV-1 activity of compound is shown in Table 1.

4.5. Anti-HIV-1 Assay. Cytotoxicity against C8166 cells $\left(\mathrm{CC}_{50}\right)$ was assessed using the MTT method, and antiHIV-1 activity was evaluated by the inhibition assay for the cytopathic effects of HIV-1(EC 50 [20].

4.6. Molecular Structure. The equilibrium geometry optimization of "Karanjin" has been achieved by energy minimization, using DFT at the B3LYP level, employing LANL2DZ as the basis set given in Table 2. The optimized geometry of the molecule under study is confirmed to be located at the local true minima on potential energy surface, as the calculated vibrational spectra contain no imaginary wavenumber. "Karanjin" is an unsymmetrical molecule having $C_{1}$ point group symmetry. The given molecule has four rings. Out of these, three are six membered hexagonal rings and one five membered pentagonal ring in which $\mathrm{A}$ and $\mathrm{C}$ are heterocyclic rings in which one carbon is replaced by oxygen. Due to the antibonding repulsion, these rings are slightly shifted towards the plane. The given structure of "Karanjin" is slightly shifted from the planar structure to minimize its surface energy. Due to this reason, ring $\mathrm{D}$ gets shifted from its plane. The optimized bond length of $\mathrm{C}-\mathrm{C}$ in five membered ring $\mathrm{A}$ ranges between $1.353 \AA$ and $1.438 \AA$, while, for another six membered ring $\mathrm{B}$, this ranges between $1.379 \AA$ and $1.415 \AA$. The optimized bond length of $\mathrm{C}-\mathrm{C}$ in six membered ring $\mathrm{C}$ ranges between $1.367 \AA$ and $1.471 \AA$, while, for another six
TABLE 1

\begin{tabular}{lccc}
\hline Compound & $\begin{array}{c}\text { Cytotoxicity } \\
\mathrm{CC}_{50}(\mu \mathrm{M})\end{array}$ & $\begin{array}{c}\text { Anti-HIV-1 } \\
\text { activity, } \\
\mathrm{EC}_{50}(\mu \mathrm{M})\end{array}$ & $\begin{array}{c}\text { Selectivity } \\
\text { index, } \\
\mathrm{CC}_{50} / \mathrm{EC}_{50}\end{array}$ \\
\hline KJ & 693.15 & 49.43 & $>14.02$ \\
AZT & 5746.1 & 0.0147 & 390406.06 \\
\hline
\end{tabular}

membered ring $\mathrm{D}$, this ranges between $1.389 \AA$ and $1.405 \AA$. This difference in the $\mathrm{C}-\mathrm{C}$ bond length is attributed to the difference in bond strength. The optimized $\mathrm{C}-\mathrm{O}$ bond lengths in ring $\mathrm{A}$ are found to be $1.362 \AA$ and $1.373 \AA$, while, in ring $\mathrm{C}$, the optimized $\mathrm{C}-\mathrm{O}$ bond lengths are found to be $1.357 \AA$ and $1.371 \AA$. The optimized C-O bond length attached to ring $\mathrm{C}$ is found to be $1.363 \AA$. Bond length of carbonyl group $\mathrm{C}=\mathrm{O}$ attached to the ring $\mathrm{C}$ is calculated to be $1.227 \AA$. Values of all the bond angles are given in Table 2 and all are in agreement with the previous experimental and theoretical studies on different biomolecules [21-23].

4.7. Vibrational Assignments. The molecule "Karanjin" contains 34 atoms and therefore has 96 normal modes of vibration. All the 96 fundamental vibrations are IR active. The harmonic vibrational frequencies calculated for Karanjin at DFT (B3LYP) level using LANL2DZ as the basis set and the experimental frequencies (FTIR) have been compared in Table 3 along with their vibrational assignments of the normal modes. Vibrational assignments are based on the observation of the animated modes in GaussView.

In "Karanjin," the $\mathrm{C}-\mathrm{H}$ functional group is present at a number of positions. The stretching vibration, $\nu(\mathrm{C}-\mathrm{H})$, is expected to occur in the region $2900-3200 \mathrm{~cm}^{-1}$. The calculated values of the $v(\mathrm{C}-\mathrm{H})$ vibration lie within this spectral range. For $\mathrm{C}-\mathrm{H}$ stretching vibrations, intense bands are calculated at 2902,2989 , and $3060 \mathrm{~cm}^{-1}$ which matches well the experimental frequencies observed at 2929, 2972, and $3052 \mathrm{~cm}^{-1}$.

The other important stretching vibrations correspond to the $\mathrm{C}=\mathrm{O}$ moieties at the $\mathrm{C}_{7}$ position. The region 1600$1750 \mathrm{~cm}^{-1}$ is generally considered as the double bond stretching region for $\mathrm{C}=\mathrm{O}, \mathrm{C}=\mathrm{C}$, and $\mathrm{C}=\mathrm{N}$ bonds [24-27]. The $\mathrm{C}=\mathrm{O}$ stretching vibration, $v(\mathrm{C}=\mathrm{O})$, appears as a prominent mode in the FTIR spectra at $1624 \mathrm{~cm}^{-1}$ which matches well the calculated one, that is, $1632 \mathrm{~cm}^{-1}$. For C-C stretching vibration an intense band is calculated at $1539 \mathrm{~cm}^{-1}$ which is found to be in good agreement with the experimental one, that is, $1526 \mathrm{~cm}^{-1}$. Due to the deformation of ring $\mathrm{A}$ vibration, an intense band is calculated at $1369 \mathrm{~cm}^{-1}$ which is in very good agreement with the experimental one, that is, $1369 \mathrm{~cm}^{-1}$. Due to breathing mode in ring B vibration, intense band is calculated at $1250 \mathrm{~cm}^{-1}$ which nearly matches the experimental one, that is, $1225 \mathrm{~cm}^{-1}$. Due to out of plane $(\mathrm{C}-\mathrm{C}-\mathrm{H})$ vibration, intense band appears at $739 \mathrm{~cm}^{-1}$. The $-\mathrm{CH}_{3}$ functional group is an important constituent of "Karanjin" and vibrations corresponding to this group are present in a number of modes. The stretching vibrations of these groups appear in a number of modes. An intense band due to butterfly motion in $\mathrm{CH}_{3}$ appears in the experimental 
TABLE 2: Optimized geometrical parameters of Karanjin by B3LYP/6-311G (d, p) methods.

\begin{tabular}{|c|c|c|c|c|c|c|}
\hline S. number & Bond lengths & Calculated & Exp. & Bond angles & Calculated & Exp. \\
\hline 1 & $R(1,2)$ & 1.3795 & 1.363 & $A(2,1,6)$ & 116.5065 & 116.5 \\
\hline 2 & $R(1,6)$ & 1.3975 & 1.399 & $A(2,1,28)$ & 122.364 & - \\
\hline 3 & $R(1,28)$ & 1.0821 & - & $A(6,1,28)$ & 121.1293 & - \\
\hline 4 & $R(2,3)$ & 1.4157 & 1.403 & $A(1,2,3)$ & 121.6696 & 121.6 \\
\hline 5 & $R(2,27)$ & 1.0826 & - & $A(1,2,27)$ & 121.4181 & - \\
\hline 6 & $R(3,4)$ & 1.3939 & 1.384 & $A(3,2,27)$ & 116.9123 & - \\
\hline 7 & $R(3,7)$ & 1.4717 & 1.466 & $A(2,3,4)$ & 120.0707 & 120.9 \\
\hline 8 & $R(4,5)$ & 1.4039 & 1.411 & $A(2,3,7)$ & 120.5299 & 121.4 \\
\hline 9 & $R(4,10)$ & 1.3573 & 1.352 & $A(4,3,7)$ & 119.3901 & 117.8 \\
\hline 10 & $R(5,6)$ & 1.3991 & 1.374 & $A(3,4,5)$ & 119.8451 & 118.8 \\
\hline 11 & $R(5,24)$ & 1.4389 & 1.431 & $A(3,4,10)$ & 122.4041 & 124.0 \\
\hline 12 & $R(6,26)$ & 1.3621 & 1.355 & $A(5,4,10)$ & 117.7498 & 117.2 \\
\hline 13 & $R(7,9)$ & 1.4724 & 1.443 & $A(4,5,6)$ & 117.6428 & 117.9 \\
\hline 14 & $R(7,11)$ & 1.2273 & 1.238 & $A(4,5,24)$ & 136.2417 & 135.7 \\
\hline 15 & $R(8,9)$ & 1.3672 & 1.353 & $A(6,5,24)$ & 106.1152 & 106.3 \\
\hline 16 & $R(8,10)$ & 1.3712 & 1.366 & $A(1,6,5)$ & 124.264 & 124.4 \\
\hline 17 & $R(8,13)$ & 1.4743 & 1.474 & $A(1,6,26)$ & 125.9683 & 125.1 \\
\hline 18 & $R(9,12)$ & 1.3632 & 1.365 & $A(5,6,26)$ & 109.7673 & 110.5 \\
\hline 19 & $R(12,31)$ & 1.4406 & 1.454 & $A(3,7,9)$ & 114.5711 & 115.3 \\
\hline 20 & $R(13,14)$ & 1.4043 & 1.395 & $A(3,7,11)$ & 122.8728 & 122.5 \\
\hline 21 & $R(13,18)$ & 1.4055 & 1.389 & $A(9,7,11)$ & 122.5555 & 122.1 \\
\hline 22 & $R(14,15)$ & 1.391 & 1.388 & $A(9,8,10)$ & 120.8623 & 120.9 \\
\hline 23 & $R(14,19)$ & 1.0793 & - & $A(9,8,13)$ & 127.8731 & 127.8 \\
\hline 24 & $R(15,16)$ & 1.3929 & 1.390 & $A(10,8,13)$ & 111.2639 & 111.3 \\
\hline 25 & $R(15,20)$ & 1.0841 & - & $A(7,9,8)$ & 121.7683 & 122.4 \\
\hline 26 & $R(16,17)$ & 1.3936 & 1.367 & $A(7,9,12)$ & 119.0806 & 117.7 \\
\hline 27 & $R(16,21)$ & 1.0841 & - & $A(8,9,12)$ & 119.0731 & 119.8 \\
\hline 28 & $R(17,18)$ & 1.3894 & 1.388 & $A(4,10,8)$ & 120.97 & 119.6 \\
\hline 29 & $R(17,22)$ & 1.0842 & - & $A(9,12,31)$ & 116.681 & 113.6 \\
\hline 30 & $R(18,23)$ & 1.0818 & - & $A(8,13,14)$ & 122.193 & 121.2 \\
\hline 31 & $R(24,25)$ & 1.3531 & 1.335 & $A(8,13,18)$ & 119.157 & 120.1 \\
\hline 32 & $R(24,29)$ & 1.0773 & - & $A(14,13,18)$ & 118.6442 & 118.7 \\
\hline 33 & $R(25,26)$ & 1.3737 & 1.377 & $A(13,14,15)$ & 120.2939 & 121.0 \\
\hline 34 & $R(25,30)$ & 1.0771 & - & $A(13,14,19)$ & 119.5231 & - \\
\hline 35 & $R(31,32)$ & 1.0891 & - & $A(15,14,19)$ & 120.1826 & - \\
\hline 36 & $R(31,33)$ & 1.0884 & - & $A(14,15,16)$ & 120.6002 & 119.1 \\
\hline 37 & $R(31,34)$ & 1.0949 & - & $A(14,15,20)$ & 119.3481 & - \\
\hline 38 & - & - & - & $A(16,15,20)$ & 120.051 & - \\
\hline 39 & - & - & - & $A(15,16,17)$ & 119.5338 & 120.1 \\
\hline 40 & - & - & - & $A(15,16,21)$ & 120.2524 & - \\
\hline 41 & - & - & - & $A(17,16,21)$ & 120.2136 & - \\
\hline 42 & - & - & - & $A(16,17,18)$ & 120.2358 & 120.9 \\
\hline 43 & - & - & - & $A(16,17,22)$ & 120.136 & - \\
\hline 44 & - & - & - & $A(18,17,22)$ & 119.6282 & - \\
\hline 45 & - & - & - & $A(13,18,17)$ & 120.6874 & 120.0 \\
\hline 46 & - & - & - & $A(13,18,23)$ & 119.4489 & - \\
\hline 47 & - & - & - & $A(17,18,23)$ & 119.8616 & - \\
\hline 48 & - & - & - & $A(5,24,25)$ & 105.5988 & 105.5 \\
\hline 49 & - & - & - & $A(5,24,29)$ & 127.7368 & - \\
\hline 50 & - & - & - & $A(25,24,29)$ & 126.6633 & - \\
\hline 51 & - & - & - & $A(24,25,26)$ & 112.0571 & 112.3 \\
\hline
\end{tabular}


TABLe 2: Continued.

\begin{tabular}{|c|c|c|c|c|c|c|}
\hline S. number & Bond lengths & Calculated & Exp. & Bond angles & Calculated & Exp. \\
\hline 52 & - & - & - & $A(24,25,30)$ & 132.815 & - \\
\hline 53 & - & - & - & $A(26,25,30)$ & 115.1279 & - \\
\hline 54 & - & - & - & $A(6,26,25)$ & 106.4614 & 105.4 \\
\hline 55 & - & - & - & $A(12,31,32)$ & 105.499 & - \\
\hline 56 & - & - & - & $A(12,31,33)$ & 110.6157 & - \\
\hline 57 & - & - & - & $A(12,31,34)$ & 110.2584 & - \\
\hline 58 & - & - & - & $A(32,31,33)$ & 110.4607 & - \\
\hline 59 & - & - & - & $A(32,31,34)$ & 109.3804 & - \\
\hline 60 & - & - & - & $A(33,31,34)$ & 110.5095 & - \\
\hline
\end{tabular}

TABLE 3: Vibrational assignments of Karanjin with B3LYP/6-311G (d, p).

\begin{tabular}{|c|c|c|c|}
\hline B3LYP (calculate) & IR (int.) & Exp. & Vibrational assignments \\
\hline 41 & 0.4616 & - & Ring D twist from rest of the molecule \\
\hline 47 & 0.0408 & - & Slight bending in whole molecule \\
\hline 58 & 1.4783 & - & Slight bending in whole molecule \\
\hline 71 & 1.2198 & - & Rock $\mathrm{CH}_{3}$ \\
\hline 86 & 1.3868 & - & Slight bending in whole molecule \\
\hline 99 & 1.2684 & - & Twist $\mathrm{CH}_{3}$ \\
\hline 139 & 1.8339 & - & Twist $\mathrm{CH}_{3}$ \\
\hline 165 & 0.5168 & - & Twist $\mathrm{CH}_{3}$ \\
\hline 182 & 1.3627 & - & Bending in whole molecule \\
\hline 216 & 3.8318 & - & Floating of whole molecule \\
\hline 231 & 1.4454 & - & Bending in whole molecule \\
\hline 257 & 4.7668 & - & $\gamma(\mathrm{C}-\mathrm{C}-\mathrm{C})$ in whole molecule \\
\hline 265 & 0.8668 & - & Whole molecule stretching \\
\hline 299 & 1.2843 & - & $\gamma(\mathrm{C}-\mathrm{C}-\mathrm{C})$ in whole molecule \\
\hline 314 & 3.3777 & - & Twist $\left(\mathrm{C}-\mathrm{O}-\mathrm{CH}_{3}\right)$ \\
\hline 324 & 4.4912 & - & $\tau(\mathrm{C}-\mathrm{C}-\mathrm{C}=\mathrm{O})$ \\
\hline 362 & 1.0152 & - & $\tau\left(\mathrm{C}-\mathrm{C}-\mathrm{O}-\mathrm{CH}_{3}\right)$ \\
\hline 398 & 0.2783 & - & $\gamma(\mathrm{C}-\mathrm{C}-\mathrm{C})$ Ring $\mathrm{D}$ \\
\hline 412 & 16.6519 & 422 & Ring A bends from joint to ring $B$ \\
\hline 441 & 1.5627 & - & $\gamma(\mathrm{C}-\mathrm{C}-\mathrm{C})$ ring $\mathrm{D}$ \\
\hline 477 & 10.432 & - & $\tau(\mathrm{C}-\mathrm{C}-\mathrm{C}-\mathrm{C})$ in whole molecule \\
\hline 486 & 4.307 & 490 & $\tau\left(\mathrm{C}-\mathrm{C}-\mathrm{O}-\mathrm{CH}_{3}\right)$ \\
\hline 525 & 0.5385 & - & $\gamma(\mathrm{C}-\mathrm{C}-\mathrm{H})$ rings $\mathrm{A}$ and $\mathrm{B}$ \\
\hline 549 & 1.0232 & - & $\tau(\mathrm{C}-\mathrm{C}-\mathrm{C}-\mathrm{C})$ ring $\mathrm{B}$ \\
\hline 580 & 5.7233 & 589 & $\gamma(\mathrm{C}-\mathrm{C}-\mathrm{H})$ ring $\mathrm{A}$ \\
\hline 607 & 1.0343 & - & $\tau(\mathrm{C}-\mathrm{C}-\mathrm{C}-\mathrm{C})$ ring $\mathrm{D}$ \\
\hline 615 & 5.088 & - & $\tau(\mathrm{C}-\mathrm{C}-\mathrm{C}-\mathrm{C})$ ring $\mathrm{D}$ \\
\hline 622 & 5.8291 & - & $\tau(\mathrm{C}-\mathrm{C}-\mathrm{C}-\mathrm{C})$ ring $\mathrm{D}$ \\
\hline 629 & 7.1766 & 632 & $\gamma(\mathrm{C}-\mathrm{C}-\mathrm{C})$ ring $\mathrm{D}+\gamma(\mathrm{C}-\mathrm{C}-\mathrm{H})$ ring $\mathrm{D}$ \\
\hline 639 & 2.7418 & - & $\tau(\mathrm{C}-\mathrm{C}-\mathrm{C}-\mathrm{O})+\tau(\mathrm{C}-\mathrm{C}-\mathrm{C}-\mathrm{C})$ \\
\hline 673 & 15.257 & - & $\beta(C-C-C)$ ring $B$ \\
\hline 681 & 35.221 & 693 & $\gamma(\mathrm{C}-\mathrm{C}-\mathrm{H})$ ring $\mathrm{D}$ \\
\hline 714 & 5.4099 & - & $\gamma(\mathrm{C}-\mathrm{C}-\mathrm{H})$ ring $\mathrm{A}$ \\
\hline 739 & 70.1363 & 730 & $\gamma(\mathrm{C}-\mathrm{C}-\mathrm{H})$ ring $\mathrm{A}$ \\
\hline 743 & 19.7507 & - & Bending in whole molecule \\
\hline 757 & 18.7847 & 757 & $\gamma(\mathrm{C}-\mathrm{C}-\mathrm{H})$ ring $\mathrm{D}+\gamma(\mathrm{C}-\mathrm{C}-\mathrm{C})$ ring $\mathrm{D}$ \\
\hline 773 & 7.1237 & - & $\gamma(\mathrm{C}-\mathrm{C}-\mathrm{H})$ in whole molecule \\
\hline
\end{tabular}


TABle 3: Continued.

\begin{tabular}{|c|c|c|c|}
\hline B3LYP (calculate) & IR (int.) & Exp. & Vibrational assignments \\
\hline 809 & 11.2216 & - & $\gamma(\mathrm{C}-\mathrm{C}-\mathrm{H})$ ring $\mathrm{B}$ \\
\hline 819 & 9.2691 & - & $\beta(C-C-C)$ ring $\mathrm{B}+\beta(C-O-C)$ ring $A$ \\
\hline 825 & 1.1522 & - & $\gamma(\mathrm{C}-\mathrm{C}-\mathrm{H})$ ring $\mathrm{D}$ \\
\hline 839 & 0.8883 & 833 & $\gamma(\mathrm{C}-\mathrm{C}-\mathrm{H})$ ring $\mathrm{A}$ \\
\hline 870 & 12.1445 & 886 & $\beta(\mathrm{C}-\mathrm{C}-\mathrm{O})$ ring $\mathrm{A}+\beta(\mathrm{C}-\mathrm{C}-\mathrm{C})$ ring $\mathrm{A}$ \\
\hline 907 & 1.2768 & - & $\gamma(\mathrm{C}-\mathrm{C}-\mathrm{H})$ ring $\mathrm{D}$ \\
\hline 922 & 9.1487 & - & $\beta(C-C-C)$ rings $C$ and $D$ \\
\hline 935 & 16.2356 & - & $\beta(\mathrm{C}-\mathrm{C}-\mathrm{H})$ ring $\mathrm{A}+\beta(\mathrm{C}-\mathrm{C}-\mathrm{C})$ ring $\mathrm{B}$ \\
\hline 945 & 0.0615 & - & $\gamma(\mathrm{C}-\mathrm{C}-\mathrm{H})$ ring $\mathrm{B}$ \\
\hline 951 & 1.3957 & 954 & $\gamma(\mathrm{C}-\mathrm{C}-\mathrm{H})$ ring $\mathrm{D}$ \\
\hline 968 & 0.4403 & - & $\gamma(\mathrm{C}-\mathrm{C}-\mathrm{H})$ ring $\mathrm{D}$ \\
\hline 976 & 0.873 & - & $\beta(C-C-C)$ ring $D$ \\
\hline 1002 & 24.0341 & - & $\omega(\mathrm{O}-\mathrm{H})$ \\
\hline 1006 & 14.4736 & - & $\beta(\mathrm{C}-\mathrm{C}-\mathrm{H})$ rings $\mathrm{A}$ and $\mathrm{B}+\beta(\mathrm{C}-\mathrm{C}-\mathrm{C})$ ring $\mathrm{B}$ \\
\hline 1014 & 24.7318 & - & $\beta(\mathrm{C}-\mathrm{C}-\mathrm{H})$ ring $\mathrm{D}$ \\
\hline 1034 & 37.6874 & 1032 & $\beta(\mathrm{C}-\mathrm{C}-\mathrm{O})$ ring $\mathrm{A}+\beta(\mathrm{C}-\mathrm{C}-\mathrm{H})$ ring $\mathrm{A}$ \\
\hline 1065 & 37.523 & 1078 & $\beta(\mathrm{C}-\mathrm{C}-\mathrm{H})$ ring $\mathrm{D}$ \\
\hline 1108 & 32.1303 & - & $\beta(\mathrm{C}-\mathrm{C}-\mathrm{H})$ rings $\mathrm{A}$ and $\mathrm{B}$ \\
\hline 1112 & 3.026 & - & $\beta(\mathrm{C}-\mathrm{C}-\mathrm{H})$ rings $\mathrm{A}$ and $\mathrm{B}$ \\
\hline 1120 & 14.9274 & - & Twist $\mathrm{CH}_{3}$ \\
\hline 1138 & 3.8577 & - & $\beta(\mathrm{C}-\mathrm{C}-\mathrm{H})$ ring $\mathrm{D}$ \\
\hline 1140 & 195.8791 & 1132 & $\beta(\mathrm{C}-\mathrm{C}-\mathrm{H})$ in whole molecule $+\beta(\mathrm{C}-\mathrm{C}-\mathrm{C})$ ring $\mathrm{B}$ \\
\hline 1151 & 51.793 & & Twist $\mathrm{CH}_{3}+\beta(\mathrm{C}-\mathrm{C}-\mathrm{H})$ in whole molecule \\
\hline 1164 & 11.6598 & 1163 & $\beta(\mathrm{C}-\mathrm{C}-\mathrm{H})$ ring $\mathrm{D}$ \\
\hline 1187 & 103.9113 & - & $\beta(\mathrm{C}-\mathrm{C}-\mathrm{H})$ in whole molecule \\
\hline 1195 & 103.0799 & - & $\beta(\mathrm{C}-\mathrm{C}-\mathrm{H})$ ring $\mathrm{B}+$ twist $\mathrm{CH}_{3}$ \\
\hline 1214 & 5.214 & - & $\beta(\mathrm{C}-\mathrm{C}-\mathrm{H})$ rings $\mathrm{A}$ and $\mathrm{B}$ \\
\hline 1250 & 100.8387 & 1225 & Breathing in ring $B$ \\
\hline 1273 & 8.6432 & - & Ring D deformation \\
\hline 1304 & 6.3433 & - & $\beta(\mathrm{C}-\mathrm{C}-\mathrm{H})$ ring $\mathrm{D}$ \\
\hline 1310 & 19.154 & - & $\beta(\mathrm{C}-\mathrm{C}-\mathrm{H})$ in whole molecule \\
\hline 1330 & 153.5339 & 1339 & $\beta(\mathrm{C}-\mathrm{C}-\mathrm{C})$ rings $\mathrm{B}$ and $\mathrm{C}+\beta(\mathrm{C}-\mathrm{C}-\mathrm{H})$ ring $\mathrm{D}$ \\
\hline 1369 & 121.7396 & 1369 & Ring A deformation \\
\hline 1408 & 52.4486 & 1405 & Butterfly in $\mathrm{CH}_{3}$ \\
\hline 1417 & 8.0248 & - & $\beta(\mathrm{C}-\mathrm{C}-\mathrm{H})$ in whole molecule \\
\hline 1418 & 17.7601 & - & $\beta(\mathrm{C}-\mathrm{C}-\mathrm{H})$ in whole molecule \\
\hline 1424 & 2.3616 & - & $\mathrm{S}(\mathrm{H}-\mathrm{C}-\mathrm{H})$ in $\mathrm{CH}_{3}$ \\
\hline 1434 & 72.9917 & - & $\beta(\mathrm{C}-\mathrm{C}-\mathrm{H})$ ring $\mathrm{B}+\nu(\mathrm{C}-\mathrm{C})$ ring $\mathrm{A}$ \\
\hline 1456 & 17.1709 & - & $S(\mathrm{H}-\mathrm{C}-\mathrm{H})$ in $\mathrm{CH}_{3}$ \\
\hline 1465 & 14.0125 & 1460 & $\beta(\mathrm{C}-\mathrm{C}-\mathrm{H})$ ring $\mathrm{D}$ \\
\hline 1504 & 14.0743 & - & $\nu(\mathrm{C}-\mathrm{C})$ ring $\mathrm{A}$ \\
\hline 1539 & 26.0649 & 1526 & $\nu(\mathrm{C}-\mathrm{C})$ rings $\mathrm{C}$ and $\mathrm{D}$ \\
\hline 1554 & 6.2017 & - & $\nu(\mathrm{C}-\mathrm{C})$ in whole molecule \\
\hline 1566 & 74.9616 & - & $\nu(\mathrm{C}-\mathrm{C})$ in whole molecule \\
\hline 1578 & 1.1856 & - & $\nu(\mathrm{C}-\mathrm{C}) \operatorname{ring} \mathrm{D}$ \\
\hline 1592 & 64.7197 & - & Ring A deformation \\
\hline 1632 & 379.2331 & 1624 & $v(\mathrm{C}=\mathrm{O})$ \\
\hline 2902 & 62.4466 & 2929 & $v(\mathrm{C}-\mathrm{H})$ in $\left(\mathrm{O}-\mathrm{CH}_{3}\right)$ \\
\hline 2989 & 35.857 & 2972 & $v(\mathrm{C}-\mathrm{H})$ in $\left(\mathrm{O}-\mathrm{CH}_{3}\right)$ \\
\hline 3024 & 7.4574 & - & $v(\mathrm{C}-\mathrm{H})$ in $\left(\mathrm{O}-\mathrm{CH}_{3}\right)$ \\
\hline
\end{tabular}


TABLE 3: Continued.

\begin{tabular}{lccc}
\hline B3LYP (calculate) & IR (int.) & Exp. & Vibrational assignments \\
\hline 3038 & 0.0903 & - & $\nu(\mathrm{C}-\mathrm{H})$ ring D \\
3048 & 14.3705 & - & $\nu(\mathrm{C}-\mathrm{H})$ ring D \\
3060 & 29.321 & 3052 & $\nu(\mathrm{C}-\mathrm{H})$ ring D \\
3072 & 1.1549 & - & $\nu(\mathrm{C}-\mathrm{H})$ ring B \\
3078 & 5.8476 & - & $\nu(\mathrm{C}-\mathrm{H})$ ring D \\
3084 & 4.8586 & - & $\nu(\mathrm{C}-\mathrm{H})$ ring B \\
3106 & 2.4619 & - & $\nu(\mathrm{C}-\mathrm{H})$ ring D \\
3127 & 1.4105 & 3131 & $\nu(\mathrm{C}-\mathrm{H})$ ring A \\
3149 & 0.1629 & 3153 & $\nu(\mathrm{C}-\mathrm{H})$ ring A \\
\hline
\end{tabular}

$\nu$ : stretching; $\beta$ : in plane bending; $\gamma$ : out of plane bending; $\tau$ : torsion.

TABLE 4: Calculated parameters using TDDFT//B3LYP/LANL2DZ for Karanjin.

\begin{tabular}{|c|c|c|c|c|}
\hline Excitation & $\begin{array}{c}\text { CI } \\
\text { coefficient }\end{array}$ & $\begin{array}{l}\text { Expansion wave length (nm) } \\
\text { calculated (Exp.) }\end{array}$ & Oscillator strength & Energy $(\mathrm{eV})$ \\
\hline \multicolumn{5}{|c|}{ Excited state 1} \\
\hline $74 \rightarrow 77$ & 0.29364 & \multirow{2}{*}{$293.89(310)$} & \multirow{2}{*}{0.2818} & \multirow{2}{*}{4.2188} \\
\hline $75 \rightarrow 77$ & 0.59270 & & & \\
\hline \multicolumn{5}{|c|}{ Excited state 2} \\
\hline $73 \rightarrow 78$ & 0.38454 & \multirow{3}{*}{$209.02(274)$} & \multirow{3}{*}{0.3189} & \multirow{3}{*}{5.9317} \\
\hline $75 \rightarrow 79$ & 0.28540 & & & \\
\hline $76 \rightarrow 81$ & 0.35123 & & & \\
\hline \multicolumn{5}{|c|}{ Excited state 3} \\
\hline $69 \rightarrow 78$ & 0.29225 & \multirow{3}{*}{182.47} & \multirow{3}{*}{0.2131} & \multirow{3}{*}{6.7948} \\
\hline $70 \rightarrow 79$ & 0.37746 & & & \\
\hline $72 \rightarrow 80$ & 0.37599 & & & \\
\hline
\end{tabular}

spectrum at $1405 \mathrm{~cm}^{-1}$ which matches well the peak at $1408 \mathrm{~cm}^{-1}$, in the calculated spectrum.

In "Karanjin," a very important vibration corresponds to the modes involving the vibrations of the ring atoms. For the purpose of simplifying the analysis, we have classified the structure of "Karanjin" into four rings A, B, C, and D as shown in Figure 1. The ring stretching vibrations, $v$ (ring), are complicated combinations of the stretching of $\mathrm{C}-\mathrm{O}$ and $\mathrm{C}-$ $\mathrm{C}$ bonds. The most important ring stretching vibrations are the ring breathing, ring deformation, and so forth. Other ring vibration modes present a mixed profile.

There are some frequencies in the lower region due to the torsion and mixed bending modes having appreciable IR intensity in calculated FTIR spectrum. Furthermore, the study of low frequency vibrations is of great significance, because it gives information on weak intermolecular interactions, which takes place in enzyme reactions [28]. Knowledge of low frequency mode is also essential for the interpretation of the effect of electromagnetic radiation on biological systems [29].

The calculated (scaled) and experimental frequencies show some deviation which can be due to the combination of electron correlation effects, insufficiency of basis set, and the unevenness of the potential energy surface and also may be explained by the presence of external medium taken during experimental FTIR analysis. The theoretical calculations have been done on gas-phase molecule.
4.8. Electronic Spectra and Electronic Properties of Karanjin. On the basis of fully optimized ground-state structure, TDDFT//B3LYP/LANL2DZ calculations have been used to determine the low-lying excited states of "Karanjin." The parameters calculated involve the vertical excitation energies, oscillator strength $(f)$, and wavelength by using the Gaussian 09W code. Experimental wavelengths are not available so these calculated data can presumably help the experimentalists. Electronic transitions determined from excited state calculations are listed in Table 4 for the three lowest energy transitions of the molecule. TD-DFT calculation predicts three intense electronic transitions at $4.2188 \mathrm{eV}(293.89 \mathrm{~nm})$, $5.9317 \mathrm{eV}$ (209.02), and 6.7948 eV (182.47) with an oscillator strengths of $0.2818,0.3189$, and 0.2131 , respectively, which are compared with the measured experimental data (Exp. $=$ $310 \mathrm{~nm}$ and $274 \mathrm{~nm}$ ).

The electronic structure of the "Karanjin" in the gas phase has been calculated with DFT using the B3LYP /6-311 G (d, p) as the basis set. HOMO and LUMO are the basic electronic parameters associated with the orbital in a molecule and the difference between them, resulting in energy gap. Not only energy gap (frontier orbital gap) helps to describe the chemical reactivity and kinetic stability of the molecule but also these orbitals find out the way the molecule interacts with other species. The HOMO-LUMO energy gap is an important measure for stability index. It establishes correlations in various chemical and biochemical systems [30, 31]. 

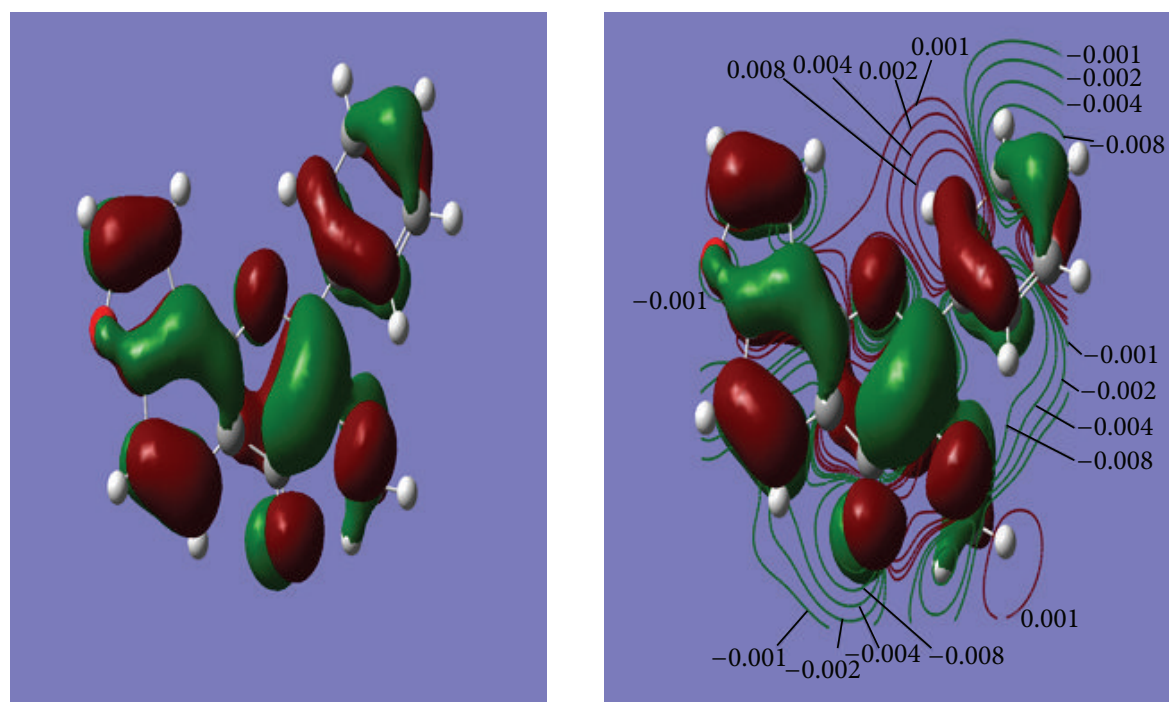

FIgURE 4: 3D and 2D plots of the highest occupied molecular orbital for Karanjin.
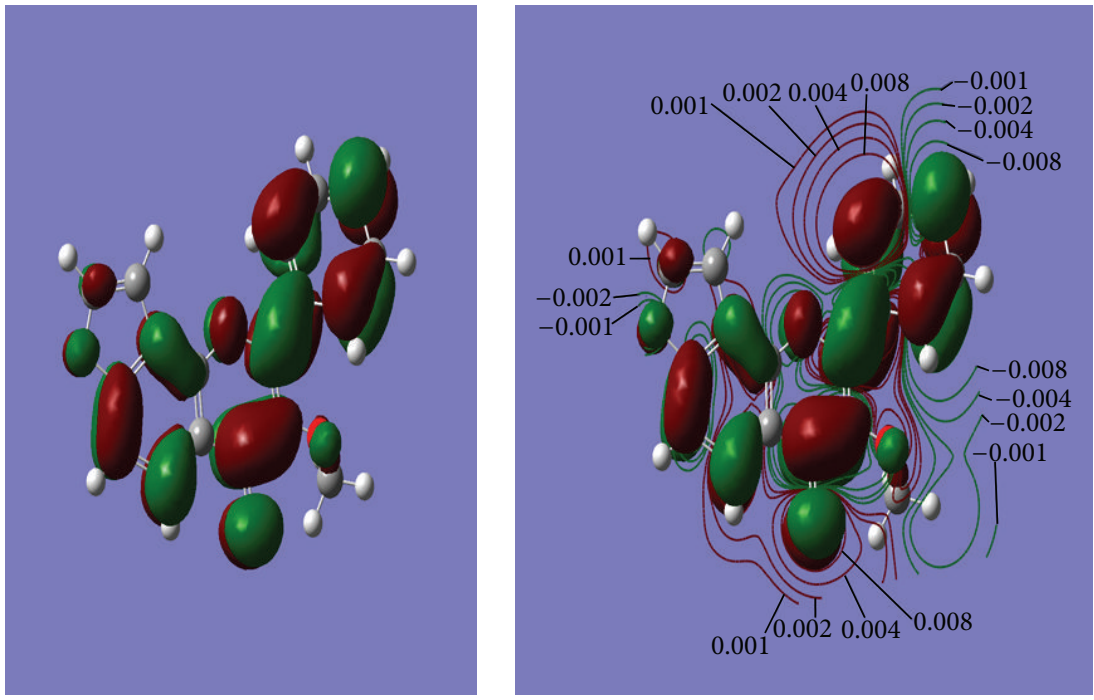

Figure 5: 3D and 2D plots of lowest unoccupied molecular orbital for Karanjin.

TABLE 5: Lowest energy, HOMO-LUMO gap (frontier orbital energy gap), and dipole moment of Karanjin by B3LYP/6-311G (d, p) methods.

\begin{tabular}{lc}
\hline Parameters & Karanjin \\
\hline Energy (in au) & -994.2536 \\
HOMO (in eV) & -6.17377 \\
LUMO (in eV) & -1.92332 \\
Frontier orbital energy gap (in eV) & 4.25045 \\
Dipole moment (in Debye) & 3.86 \\
\hline
\end{tabular}

The plots of the HOMO, LUMO, and electrostatic potential for both the molecules in $2 \mathrm{D}$ and $3 \mathrm{D}$ are shown in Figures 4,5 , and 6. The HOMO is found to be concentrated over the whole atoms, but the LUMO lies mainly over the whole molecule but less over ring A. The calculated value of the frontier orbital energy gap is $4.25 \mathrm{eV}$ (Table 5). The low frontier orbital gap is also associated with a high chemical reactivity and low kinetic stability [32]. The molecular electrostatic potential (MESP) is an important factor by which we can confirm the electrostatic potential region distribution of size and shape of molecules as well as the total physiology of the molecules. We have plotted 2D and 3D MESP structures of the title compound as shown in Figure 6. The electronegative region is outside the molecule near the oxygen atoms. The energy equal to the shielded potential energy surface is required for any substitution reaction near oxygen. The electronegative lines (in between -0.08 a.u. and -0.02 a.u.) form a closed contour which clearly indicates that total flux passing in between these curves is not equal to zero. For any nucleophilic substitution reaction near oxygen (closed contour area), an amount of energy equal to shielded potential energy surface is required; however, remaining part of molecule is suitable for electrophilic substitution reaction. Thus, it can be 
TABLE 6: Calculated $\varepsilon_{\text {HOMO }}, \varepsilon_{\text {LUMO }}$, energy band gap $\left(\varepsilon_{L}-\varepsilon_{H}\right)$, chemical potential $(\mu)$, electronegativity $(\chi)$, global hardness $(\eta)$, global softness $(S)$, and global electrophilicity index $(\omega)$ for Karanjin at B3LYP/6-311G (d, p) level.

\begin{tabular}{|c|c|c|c|c|c|c|c|c|}
\hline Karanjin & $\varepsilon_{H}$ & $\varepsilon_{L}$ & $\varepsilon_{L}-\varepsilon_{H}$ & $\chi$ & $\mu$ & $\eta$ & S & $\omega$ \\
\hline A & -6.17377 & -1.92332 & 4.25045 & 4.04854 & -4.04854 & 2.12523 & 0.23527 & 3.85622 \\
\hline
\end{tabular}
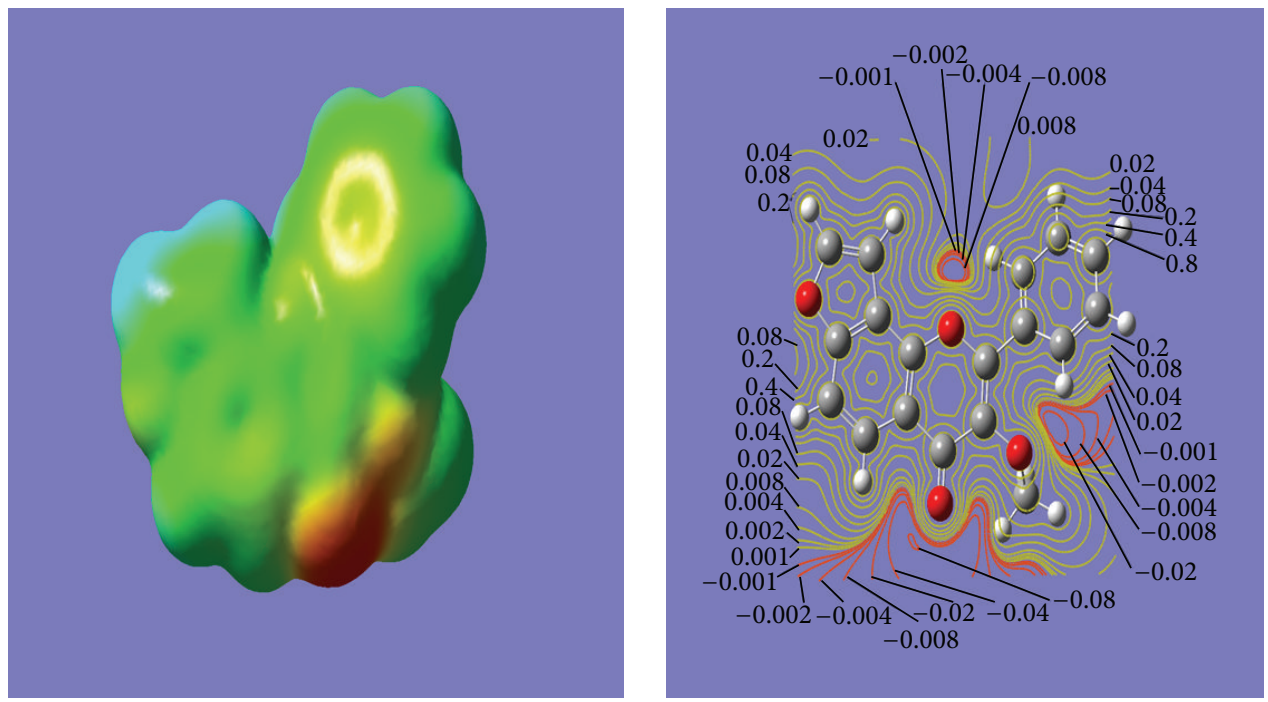

Figure 6: 3D and 2D plots of molecular electrostatic potential.

asserted that MESP values have been shown to be well related to biological properties [33-35].

4.9. Global Reactivity Descriptors. The energies of frontier molecular orbitals $\left(\varepsilon_{\mathrm{HOMO}}, \varepsilon_{\mathrm{LUMO}}\right)$, energy band gap $\left(\varepsilon_{\mathrm{LUMO}}-\right.$ $\left.\varepsilon_{\mathrm{HOMO}}\right)$, electronegativity $(\chi)$, chemical potential $(\mu)$, global hardness $(\eta)$, global softness $(S)$, and global electrophilicity index $(\omega)$ [36-39] of "Karanjin" have been listed in Table 6. On the basis of $\varepsilon_{\mathrm{HOMO}}$ and $\varepsilon_{\mathrm{LUMO}}$, these parameters are calculated using (1) as given below

$$
\begin{gathered}
\chi=-\frac{1}{2}\left(\varepsilon_{\mathrm{LUMO}}+\varepsilon_{\mathrm{HOMO}}\right) \\
\mu=-\chi=\frac{1}{2}\left(\varepsilon_{\mathrm{LUMO}}+\varepsilon_{\mathrm{HOMO}}\right) \\
\eta=\frac{1}{2}\left(\varepsilon_{\mathrm{LUMO}}-\varepsilon_{\mathrm{HOMO}}\right) \\
S=\frac{1}{2 \eta} \\
\omega=\frac{\mu^{2}}{2 \eta} .
\end{gathered}
$$

4.10. Local Reactivity Descriptors. The Fukui function (FF) of a molecule provides information on the reactivity. The FF successfully predicts relative site reactivities for most chemical systems and as such it provides a method for understanding and categorizing chemical reactions. The atom with the highest FF value is highly reactive when compared to the other atoms in the molecule. These values represent the qualitative descriptors of reactivity of different atoms in the molecule. Ayers and Parr [40] have elucidated that molecules tend to react where the FF is the largest when attacked by soft reagents and in places where the FF is found to be smaller when attacked by hard reagents. The use of the Fukui functions for the site selectivity of the Karanjin molecule for nucleophilic and electrophilic attacks has been made with special emphasis to the dependence of the Fukui values on the basis of B3LYP/6-311G(d, p) level of theory. Using the Mulliken atomic charges of neutral, cation, and anion, state of Karanjin, the Fukui functions $\left(f_{k}^{+}, f_{k}^{-}, f_{k}^{0}\right)$, local softness $\left(s_{k}^{+}, s_{k}^{-}, s_{k}^{0}\right)$, and local electrophilicity indices $\left(\omega_{k}^{+}, \omega_{k}^{-}, \omega_{k}^{0}\right)[37,38]$, the Fukui functions are calculated using the following (2):

$$
\begin{aligned}
f_{k}^{+} & =[q(N+1)-q(N)] \quad \text { for nucleophilic attack } \\
f_{k}^{-} & =[q(N)-q(N-1)] \quad \text { for electrophilic attack } \\
f_{k}^{0} & =\frac{1}{2}[q(N+1)+q(N-1)] \quad \text { for radical attack. }
\end{aligned}
$$

Local softness and electrophilicity indices are calculated using (3)

$$
\begin{array}{ccc}
s_{k}^{+}=S f_{k}^{+}, & s_{k}^{-}=S f_{k}^{-}, & s_{k}^{0}=S f_{k}^{0}, \\
\omega_{k}^{+}=\omega f_{k}^{+}, & \omega_{k}^{-}=\omega f_{k}^{-}, & \omega_{k}^{-}=\omega f_{k}^{-},
\end{array}
$$

where,+- , and 0 signs show nucleophilic, electrophilic, and radical attack, respectively.

The Fukui functions, local softnesses, and local electrophilicity indices for selected atomic sites in "Karanjin" have been listed in Table 7. The maximum values of all the three local electrophilic reactivity descriptors $\left(f_{k}^{+}, s_{k}^{+}, \omega_{k}^{+}\right)$at C7 and 
TABLE 7: (a) Fukui functions $\left(f_{k}^{+}, f_{k}^{-}\right)$, local softnesses $\left(s_{k}^{+}, s_{k}^{-}\right)$, and local electrophilicity indices $\left(\omega_{k}^{+}, \omega_{k}^{-}\right)$for selected atomic sites of Karanjin, using the Mulliken population analysis at B3LYP/6-311G (d, p) level. (b) (All atomic sites.)

(a)

\begin{tabular}{lcccccc}
\hline Atom number & $f_{k}^{+}$ & $f_{k}^{-}$ & $s_{k}^{+}$ & $s_{k}^{-}$ & $\omega_{k}^{+}$ & $\omega_{k}^{-}$ \\
\hline C1 & -0.0098 & 0.09366 & -0.0023 & 0.02203 & -0.0377 & 0.36116 \\
C2 & 0.09201 & -0.0089 & 0.02165 & -0.0021 & 0.35482 & -0.0344 \\
C7 & 0.09812 & -0.0179 & 0.02309 & -0.0042 & 0.37838 & -0.0692 \\
C14 & 0.02752 & 0.02195 & 0.00648 & 0.00516 & 0.10614 & 0.08463 \\
C15 & 0.00421 & 0.02187 & 0.00099 & 0.00515 & 0.01623 & 0.08434 \\
C16 & 0.06111 & 0.02641 & 0.01438 & 0.00621 & 0.23566 & 0.10185 \\
C17 & 0.00646 & 0.01716 & 0.00152 & 0.00404 & 0.02491 & 0.06616 \\
C18 & 0.044 & 0.01018 & 0.01035 & 0.0024 & 0.16969 & 0.03926 \\
C24 & -0.0138 & 0.05979 & -0.0032 & 0.01407 & -0.053 & 0.23054 \\
C25 & 0.05104 & 0.03085 & 0.01201 & 0.00726 & 0.19682 & 0.11898 \\
C31 & -0.1047 & 0.06052 & -0.0246 & 0.01424 & -0.4038 & 0.23338 \\
\hline
\end{tabular}

(b)

\begin{tabular}{|c|c|c|c|c|c|c|c|c|c|c|c|c|}
\hline Atom number & $M$ & $M^{-}$ & $M^{+}$ & $f_{k}^{+}$ & $f_{k}^{-}$ & $f_{k}^{0}$ & $s_{k}^{+}$ & $s_{k}^{-}$ & $s_{k}^{0}$ & $\omega_{k}^{+}$ & $\omega_{k}^{-}$ & $\omega_{k}^{0}$ \\
\hline $\mathrm{C} 1$ & -0.1023 & -0.0925 & -0.0086 & -0.0098 & 0.09366 & 0.08389 & -0.0023 & 0.02203 & 0.01974 & -0.0377 & 0.36116 & 0.3235 \\
\hline $\mathrm{C} 2$ & 0.01541 & -0.0766 & 0.00648 & 0.09201 & -0.0089 & 0.08308 & 0.02165 & -0.0021 & 0.01955 & 0.35482 & -0.0344 & 0.32039 \\
\hline $\mathrm{C} 3$ & -0.171 & -0.1801 & -0.1926 & 0.0091 & -0.0217 & -0.0126 & 0.00214 & -0.0051 & -0.003 & 0.03511 & -0.0835 & -0.0484 \\
\hline $\mathrm{C} 4$ & 0.29134 & 0.20787 & 0.22905 & 0.08347 & -0.0623 & 0.02118 & 0.01964 & -0.0147 & 0.00498 & 0.32189 & -0.2402 & 0.08169 \\
\hline C5 & -0.1988 & -0.1379 & -0.1236 & -0.0609 & 0.07519 & 0.01429 & -0.0143 & 0.01769 & 0.00336 & -0.2348 & 0.28995 & 0.05512 \\
\hline C6 & 0.31443 & 0.18389 & 0.2206 & 0.13054 & -0.0938 & 0.03671 & 0.03071 & -0.0221 & 0.00864 & 0.5034 & -0.3618 & 0.14157 \\
\hline C7 & 0.36424 & 0.26612 & 0.3463 & 0.09812 & -0.0179 & 0.08019 & 0.02309 & -0.0042 & 0.01887 & 0.37838 & -0.0692 & 0.30921 \\
\hline $\mathrm{C} 8$ & 0.21434 & 0.15357 & 0.23516 & 0.06077 & 0.02083 & 0.0816 & 0.0143 & 0.0049 & 0.0192 & 0.23433 & 0.08032 & 0.31465 \\
\hline C9 & .01435 & 0.02251 & 0.13566 & -0.0082 & 0.12131 & 0.11315 & -0.0019 & 0.02854 & 0.02662 & -0.0314 & 0.46778 & 0.43634 \\
\hline $\mathrm{O} 10$ & -0.3576 & -0.3513 & -0.2938 & -0.0063 & 0.06379 & 0.05753 & -0.0015 & 0.01501 & 0.01354 & -0.0242 & 0.246 & 0.22185 \\
\hline O11 & -0.4282 & -0.4309 & -0.2719 & 0.00268 & 0.15627 & 0.15895 & 0.00063 & 0.03677 & 0.0374 & 0.01032 & 0.60263 & 0.61295 \\
\hline $\mathrm{O} 12$ & -0.2842 & -0.3904 & -0.3292 & 0.10616 & -0.045 & 0.06119 & 0.02498 & -0.0106 & 0.0144 & 0.40939 & -0.1734 & 0.23595 \\
\hline $\mathrm{C} 13$ & -0.1414 & -0.1257 & -0.1241 & -0.0 & 0.01735 & 0.00163 & -0.0037 & 0.00408 & 0.00038 & -0.0606 & & 0.00628 \\
\hline $\mathrm{C} 14$ & -0.039 & -0.0665 & -0.017 & 0.02752 & 0.02195 & 0.04947 & 0.00648 & 0.00516 & 0.01164 & 0.10614 & 0.08463 & 0.19077 \\
\hline $\mathrm{C} 15$ & -0.1101 & -0.1143 & -0.0882 & 0.00421 & 0.02187 & 0.02608 & 0.00099 & 0.00515 & 0.00614 & 0.01623 & 0.08434 & 0.10057 \\
\hline $\mathrm{C} 16$ & -0.0675 & -0.1286 & -0.0411 & 0.06111 & 0.02641 & 0.08752 & 0.01438 & 0.00621 & 0.02059 & 0.23566 & 0.10185 & 0.33751 \\
\hline $\mathrm{C} 17$ & -0.1018 & -0.1082 & -0.0846 & 0.00646 & 0.01716 & 0.02362 & 0.00152 & 0.00404 & 0.00556 & 491 & 0.06616 & 0.09107 \\
\hline $\mathrm{C} 18$ & -0.0203 & -0.0643 & -0.0101 & 0.044 & 0.01018 & 0.05418 & 0.01035 & 0.0024 & 0.01275 & 0.16969 & 0.03926 & 0.20895 \\
\hline H19 & 0.17629 & 0.09163 & 0.14199 & 0.08465 & -0.0343 & 0.05035 & 0.01992 & -0.0081 & 0.01185 & 0.32644 & -0.1 & 0.19418 \\
\hline $\mathrm{H} 20$ & & & & 0.04482 & & & & & & & & 0.31386 \\
\hline $\mathrm{H} 21$ & 0.09873 & 0.04495 & 0.13962 & 0.05377 & 0.0409 & 0.09467 & 0.01265 & 0.00962 & 0.02227 & 0.20735 & 0.15772 & 0.36507 \\
\hline $\mathrm{H} 22$ & 0.09777 & 0.05104 & 0.13087 & 0.04674 & 0.0331 & 0.07984 & 0.011 & 0.00779 & 0.01878 & 0.18022 & 0.12764 & 0.30787 \\
\hline $\mathrm{H} 23$ & 0.10521 & 0.07289 & 0.12009 & 0.03232 & 0.01489 & 0.04721 & 0.0076 & 0.0035 & 0.01111 & 0.12464 & 0.0574 & 0.18204 \\
\hline $\mathrm{C} 24$ & -0.109 & -0.0952 & -0.0492 & -0.0138 & 0.05979 & 0.04603 & -0.0032 & 0.01407 & 0.01083 & -0.053 & 0.23054 & 0.1775 \\
\hline $\mathrm{C} 25$ & 0.08553 & 0.03449 & 0.11639 & 0.05104 & 0.03085 & 0.08189 & 0.01201 & 0.00726 & 0.01927 & 0.19682 & 0.11898 & 0.3158 \\
\hline $\mathrm{O} 26$ & -0.3851 & -0.2927 & -0.2394 & -0.0924 & 0.1457 & 0.05331 & -0.0217 & 0.03428 & 0.01254 & -0.3563 & 0.56187 & 0.20559 \\
\hline $\mathrm{H} 27$ & & 0.07306 & & 0.05309 & 0.0249 & 0.07799 & 0.01249 & 0.00586 & 0.01835 & 0.20471 & 0.09602 & 0.30074 \\
\hline $\mathrm{H} 28$ & 0.12425 & 0.06315 & 0.15403 & 0.06109 & 0.02978 & 0.09088 & 0.01437 & 0.00701 & 0.02138 & 0.23559 & 0.11485 & 0.35044 \\
\hline $\mathrm{H} 29$ & 0.12266 & 0.08408 & 0.13502 & 0.03858 & 0.01236 & 0.05093 & 0.00908 & 0.00291 & 0.01198 & 0.14876 & 0.04765 & 0.1964 \\
\hline $\mathrm{H} 30$ & 0.1577 & 0.07866 & 0.16266 & 0.07904 & 0.00496 & 0.084 & 0.0186 & 0.00117 & 0.01976 & 0.3048 & 0.01914 & 0.32394 \\
\hline C31 & -0.202 & -0.0973 & -0.1414 & -0.1047 & 0.06052 & -0.0442 & -0.0246 & 0.01424 & -0.0104 & -0.4038 & 0.23338 & -0.1704 \\
\hline H32 & 0.07267 & 0.07634 & 0.15422 & -0.0037 & 0.08155 & 0.07788 & -0.0009 & 0.01919 & 0.01832 & -0.0142 & 0.31449 & 0.30033 \\
\hline H33 & 0.18683 & 0.12381 & 0.17165 & 0.06302 & -0.0152 & 0.04784 & 0.01483 & -0.0036 & 0.01126 & 0.24303 & -0.0585 & 0.18449 \\
\hline H34 & 0.05282 & 0.07183 & 0.1301 & -0.019 & 0.07728 & 0.05826 & -0.0045 & 0.01818 & 0.01371 & -0.0733 & 0.29799 & 0.22467 \\
\hline
\end{tabular}


TABLE 8: Polarizability and hyperpolarizability of Karanjin.

\begin{tabular}{lccr}
\hline Polarizability & Values & Hyperpolarizability & Values \\
\hline$\alpha_{X X}$ & -109.2190 & $\beta_{X X X}$ & 22.0796 \\
$\alpha_{X Y}$ & 3.9546 & $\beta_{X X Y}$ & -8.6890 \\
$\alpha_{Y Y}$ & -121.6217 & $\beta_{X Y Y}$ & -2.3092 \\
$\alpha_{Y Z}$ & 1.5294 & $\beta_{Y Y Y}$ & -61.3104 \\
$\alpha_{Z Z}$ & -127.8736 & $\beta_{X X Z}$ & -8.1237 \\
$\alpha_{X Z}$ & 0.5918 & $\beta_{X Y Z}$ & 1.2207 \\
$<\alpha>$ & 119.5714 & $\beta_{Y Y Z}$ & 22.2892 \\
- & - & $\beta_{X Z Z}$ & 1.4229 \\
- & - & $\beta_{Y Z Z}$ & 11.6211 \\
- & - & $\beta_{Z Z Z}$ & 0.2894 \\
- & - & $\beta_{\text {Total }}$ & 63.6404 \\
\hline
\end{tabular}

TABLE 9: Calculated thermodynamic properties of Karanjin by B3LYP/6-311G (d, p) methods.

\begin{tabular}{lccc}
\hline & $E($ thermal $)\left(\mathrm{kcalmol}^{-1}\right)$ & $\mathrm{CV}\left(\mathrm{cal} \mathrm{K}^{-1} \mathrm{~mol}^{-1}\right)$ & $S\left(\mathrm{cal} \mathrm{K}^{-1} \mathrm{~mol}^{-1}\right)$ \\
\hline Total & $\mathbf{1 7 1 . 7 0 0}$ & $\mathbf{6 7 . 2 4 4}$ & $\mathbf{1 3 3 . 5 3 4}$ \\
Translational & 0.889 & 2.981 & 42.913 \\
Rotational & 0.889 & 2.981 & 34.406 \\
Vibrational & 169.922 & 61.283 & 56.215 \\
\hline
\end{tabular}

C2 indicate that this site is prone to nucleophilic attack, while, for electrophilic attack, C31 and C24 are found to be the most active sites.

In pentagonal ring $\mathrm{A}$, carbon is replaced by oxygen which has the most electronegative lone pair antibonding electron which extracts electrons from the neighboring carbon having the positive charge. To cancel this positive charge, it attracts the electron from C24 carbon. So C24 provides a better electrophilic site for the soft receptors. In hexagonal ring $\mathrm{C}$, a carbon is replaced by oxygen having two lone pair antibonding electrons. Due to the repulsion of these antibonding electrons, the shape of the ring gets distorted. Ring $\mathrm{C}$ has two substituent groups at para and meta positions. At meta position, oxygen is attached to the ring $\mathrm{C}$ and at para position $\mathrm{O}-\mathrm{CH}_{3}$ group is attached. Oxygen is more electronegative than carbon which extracts electron from carbon. Due to this reason, C31 carbon atom of methyl group is a better center for electrophilic substitution. In hexagonal ring $\mathrm{C}$, electron withdrawing group $\mathrm{O}-\mathrm{CH}_{3}$ extracts electron from $\mathrm{C} 9$ atom of the ring $\mathrm{C}$ to fulfill the deficiency; $\mathrm{C} 9$ atom extracts electron from $\mathrm{C} 7$ atom and hence because of the $\mathrm{C} 7$ atom being electron deficient it extracts electron from $\mathrm{O} 11$ so $\mathrm{C} 7$ atom becomes a potential site for a nucleophilic attack.

4.11. Dipole Moment, Polarizability, Hyperpolarizability, and Thermodynamic Properties. Dipole moment $(\mu)$, polarizability $\langle\alpha\rangle$, and total first static hyperpolarizability $\beta[41,42]$ are also calculated (in Tables 5 and 8 ) by using density functional theory. They can be expressed in terms of $x, y$, and $z$ components and are given by following (4):

$$
\begin{gathered}
\mu=\left(\mu_{x}^{2}+\mu_{y}^{2}+\mu_{z}^{2}\right)^{1 / 2} \\
\langle\alpha\rangle=\frac{1}{3}\left[\alpha_{x x}+\alpha_{y y}+\alpha_{z z}\right]
\end{gathered}
$$

$$
\begin{aligned}
\beta_{\text {Total }}= & \left(\beta_{x}^{2}+\beta_{y}^{2}+\beta_{z}^{2}\right)^{1 / 2} \\
= & {\left[\left(\beta_{x x x}+\beta_{x y y}+\beta_{x z z}\right)^{2}\right.} \\
& +\left(\beta_{y y y}+\beta_{y x x}+\beta_{y z z}\right)^{2} \\
& \left.+\left(\beta_{z z z}+\beta_{z x x}+\beta_{z y y}\right)^{2}\right]^{1 / 2} .
\end{aligned}
$$

The $\beta$ components of Gaussian output are reported in atomic units, where 1 a.u. $=8.3693 \times 10^{-33}$ e.s.u. For Karanjin, the calculated dipole moment value is 3.86 Debye. Having higher dipole moment than water (2.16 Debye), "Karanjin" can be used as better solvent. We see a greater contribution of $\alpha_{z z}$ in molecule which shows that the molecule is elongated more towards $Z$ direction and is more contracted to $X$ direction. Perpendicular part contributes with a less part of polarizability of molecule. $B_{y y y}$ and $\beta_{y y z}$ contribute with a larger part of hyperpolarizability in the molecule. This shows that $Y Z$ plane and $Y$-axis are more optically active in these directions. Standard thermodynamic functions such as free energy, constant volume heat capacity CV, and entropy $S$ have also been calculated for "Karanjin" and are given in Table 9. These functions can provide helpful information for further study of the title compounds.

\section{Conclusion}

In this work, the compound "Karanjin" an anti-HIV drug was experimentally isolated and identified and its bioactivity along with detailed quantum chemical studies was carried out. The optimized geometry of the "Karanjin" molecule has been determined by the method of density functional 
theory (DFT). For both geometry and total energy, it has been combined with B3LYP functional having 6-311 $\mathrm{g}$ (d, p) as the basis set. Using this optimized structure, we have calculated the infrared wavenumbers and compared them with the experimental data. The calculated wavenumbers are in an excellent agreement with the experimental values. On the basis of fully optimized ground-state structure, TDDFT//B3LYP/LANL2DZ calculations have been used to determine the low-lying excited states of "Karanjin." Reactivity reflects the susceptibility of a substance towards a specific chemical reaction and plays a key role in, for example, the design of new molecules and understanding biological systems and material science. Hyperpolarizability is mainly controlled by the planarity of the molecules, the donor and accepter strength, and bond length alteration. The values of hyperpolarizability indicate a possible use of these compounds in electrooptical applications. We have also discussed global and local reactivity descriptors sites for both molecules during electrophilic, nucleophilic, and radical attacks. These values represent the qualitative descriptors of reactivity of different atoms in the molecule. This compound shows antiHIV activity so these theoretical and experimental aspects can provide a path for researchers in future.

\section{Conflict of Interests}

The authors of the paper have no conflict of interests in the present work.

\section{Acknowledgment}

The corresponding author Apoorva Dwivedi is grateful to Professor Neeraj Misra for providing valuable suggestions.

\section{References}

[1] The Wealth of India-A Dictionary of Indian Raw Materials, vol. 8, Council of Scientific and Industrial Research, New Delhi, India, 2005.

[2] K. R. Kirtikar and B. D. Basu, Indian Medicinal Plants, vol. 1, 2nd edition, 1981.

[3] S. A. Dahanukar, R. A. Kulkarni, and N. N. Rege, "Pharmacology of medicinal plants and natural products," Indian Journal of Pharmacology, vol. 32, no. 4, pp. S81-S118, 2000.

[4] G. P. Garg, "A new component from leaves of Pongamia glabra," Planta Medica, vol. 39, no. 1, pp. 73-74, 1979.

[5] S. B. Malik, T. R. Seshadri, and P. Sharma, "Minort components of the leaves of pongamia glabra," Indian Journal of Chemistry, vol. 14, pp. 229-230, 1976.

[6] P. Sharma, T. R. Seshadri, and S. K. Mukerjee, "Some synthesis and natural analogues of globrachromene," Indian Journal of Chemistry, vol. 11, pp. 98/5-98/6, 1973.

[7] S. Rangaswamy and T. R. Seshadri, "Extraction and recovery of karanjin: a value addition to karanja (Pongamia pinnata) seed oil," Indian Journal of Pharmacology, vol. 3, p. 3, 1941.

[8] B. S. Parmar and K. C. Gulati, "Synergists for pyrethrins (II)karanjin," Indian Journal of Entomology, vol. 31, pp. 239-243, 1969.
[9] W. E. Sapna, T. C. Sindhu Kanya, A. M. Mamatha et al., "Karanjin, a flavonoid inhibits lipoxygenases," in Proceedings of National Academy of Science India, CFTRI, Mysore, India, 2007.

[10] P. Hohenberg and W. Kohn, "Inhomogeneous electron gas," Physical Review, vol. 136, no. 3B, pp. B864-B871, 1964.

[11] A. D. Becke, "Density-functional thermochemistry. III. The role of exact exchange," The Journal of Chemical Physics, vol. 98, no. 7, pp. 5648-5652, 1993.

[12] C. Lee, W. Yang, and R. G. Parr, "Development of the ColleSalvetti correlation-energy formula into a functional of the electron density," Physical Review B, vol. 37, no. 2, pp. 785-789, 1988.

[13] M. J. Frisch, G. W. Trucks, H. B. Schlegel et al., Gaussian 09, Gaussian, Pittsburgh, Pa, USA, 2009.

[14] P. L. Fast, J. Corchado, M. L. Sanches, and D. G. Truhlar, "Optimized parameters for scaling correlation energy," The Journal of Physical Chemistry A, vol. 103, pp. 3139-3143, 1999.

[15] A. Frisch, A. B. Nelson, and A. J. Holder, "Gauss View," Pittsburgh, Pa, USA, 2005.

[16] V. Vismaya, S. M. Belagihally, S. Rajashekhar, V. B. Jayaram, S. M. Dharmesh, and S. K. C. Thirumakudalu, "Gastroprotective properties of karanjin from Karanja (Pongamia pinnata) seeds; Role as antioxidant and $\mathrm{H}+, \mathrm{K}+$-ATPase inhibitor," Evidencebased Complementary and Alternative Medicine, vol. 2011, Article ID 747246, 10 pages, 2011.

[17] S. Sharma, M. Verma, R. Prasad, and D. Yadav, "Efficacy of nonedible oil seedcakes against termite (Odontotermes obesus)," Journal of Scientific and Industrial Research, vol. 70, no. 12, pp. 1037-1041, 2011.

[18] R. Ranga Rao, A. K. Tiwari, P. Prabhakar Reddy et al., "New furanoflavanoids, intestinal $\alpha$-glucosidase inhibitory and freeradical (DPPH) scavenging, activity from antihyperglycemic root extract of Derris indica (Lam.)," Bioorganic and Medicinal Chemistry, vol. 17, no. 14, pp. 5170-5175, 2009.

[19] V. Vismaya, W. Sapna Eipeson, J. R. Manjunatha, P. Srinivas, and T. C. Sindhu Kanya, "Extraction and recovery of karanjin: a value addition to karanja (Pongamia pinnata) seed oil," Industrial Crops and Products, vol. 32, no. 2, pp. 118-122, 2010.

[20] J.-H. Wang, S.-C. Tam, H. Huang, D.-Y. Ouyang, Y.-Y. Wang, and Y.-T. Zheng, "Site-directed PEGylation of trichosanthin retained its anti-HIV activity with reduced potency in vitro," Biochemical and Biophysical Research Communications, vol. 317, no. 4, pp. 965-971, 2004.

[21] D. Sajan, H. J. Ravindra, N. Misra, and I. H. Joe, "Intramolecular charge transfer and hydrogen bonding interactions of nonlinear optical material N-benzoyl glycine: vibrational spectral study," Vibrational Spectroscopy, vol. 54, no. 1, pp. 72-80, 2010.

[22] V. Mukherjee, N. P. Singh, and R. A. Yadav, "FTIR and Raman spectra, DFT and SQMFF calculations for geometrical interpretation and vibrational analysis of some trifluorobenzoic acid dimers," Vibrational Spectroscopy, vol. 52, no. 2, pp. 163-172, 2010.

[23] M. Hariharan and S. S. Rajan, "Crystal structure communications," Acta Crystallographica C, vol. 46, pp. 437-439, 1990.

[24] M. Alcolea Palafox, G. Tardajos, A. Guerrero-Martínez et al., "FT-IR, FT-Raman spectra, density functional computations of the vibrational spectra and molecular geometry of biomolecule 5-aminouracil," Chemical Physics, vol. 340, no. 1-3, pp. 17-31, 2007.

[25] J. S. Singh, "FTIR and Raman spectra and fundamental frequencies of biomolecule: 5-Methyluracil (thymine)," Journal of Molecular Structure, vol. 876, no. 1-3, pp. 127-133, 2008. 
[26] C. P. Beetz Jr. and G. Ascarelli, "The low frequency vibrations of pyrimidine and purine bases," Spectrochimica Acta A: Molecular Spectroscopy, vol. 36, no. 3, pp. 299-313, 1980.

[27] J. Bandekar and G. Zundel, "The role of CO transition dipoledipole coupling interaction in uracil," Spectrochimica Acta A: Molecular Spectroscopy, vol. 39, no. 4, pp. 337-341, 1983.

[28] K.-C. Chou, "Biological functions of low-frequency vibrations (phonons). III. Helical structures and microenvironment," Biophysical Journal, vol. 45, no. 5, pp. 881-889, 1984.

[29] H. Frohlich, Biological Coherence and Response to External Stimuli, Springer, Berlin, Germany, 1988.

[30] D. F. V. Lewis, C. Loannides, and D. V. Parkee, "Interaction of a series of nitriles with the alcohol-inducible isoform of P450: computer analysis of structure-activity relationships," Xenobiotica, vol. 24, pp. 401-408, 1984.

[31] Z. Zhou and R. G. Parr, "Activation hardness: new index for describing the orientation of electrophilic aromatic substitution," Journal of the American Chemical Society, vol. 112, no. 15, pp. 5720-5724, 1990.

[32] I. Fleming, Frontier Orbitals and Organic Chemical Reactions, John Wiley \& Sons, New York, NY, USA, 1976.

[33] M. Bohl, K. Ponsold, and G. Reck, "Quantitative structureactivity relationships of cardiotonic steroids using empirical molecular electrostatic potentials and semiempirical molecular orbital calculations," Journal of Steroid Biochemistry, vol. 21, no. 4, pp. 373-379, 1984.

[34] D. F. Lewis and V. Griffiths, "Molecular electrostatic potential energies and methylation of DNA bases: a molecular orbitalgenerated quantitative structure-activity relationship," Xenoviotica, vol. 17, pp. 769-776, 1987.

[35] A. Kumar and P. C. Mishra, "Structure-activity relationships for some anti-HIV drugs using electric field mapping," Journal of Molecular Structure, vol. 277, pp. 299-312, 1992.

[36] R. G. Pearson, "Absolute electronegativity and hardness: applications to organic chemistry," Journal of Organic Chemistry, vol. 54, no. 6, pp. 1423-1430, 1989.

[37] R. G. Parr, L. V. Szentpály, and S. Liu, "Electrophilicity Index," Journal of the American Chemical Society, vol. 121, pp. 1922-1924, 1999.

[38] P. K. Chattaraj and S. Giri, "Stability, reactivity, and aromaticity of compounds of a multivalent superatom," Journal of Physical Chemistry A, vol. 111, no. 43, pp. 11116-11121, 2007.

[39] J. Padmanabhan, R. Parthasarathi, V. Subramanian, and P. K. Chattaraj, "Electrophilicity-based charge transfer descriptor," Journal of Physical Chemistry A, vol. 111, no. 7, pp. 1358-1361, 2007.

[40] P. W. Ayers and R. G. Parr, "Variational principles for describing chemical reactions: the Fukui function and chemical hardness revisited," Journal of the American Chemical Society, vol. 122, no. 9, pp. 2010-2018, 2000.

[41] D. A. Kleinman, "Nonlinear dielectric polarization in optical media," Physical Review B, vol. 126, no. 6, pp. 1977-1979, 1962.

[42] J. Pipek and P. G. Mezey, "A fast intrinsic localization procedure applicable for ab initio and semiempirical linear combination of atomic orbital wave functions," The Journal of Chemical Physics, vol. 90, no. 9, pp. 4916-4926, 1989. 

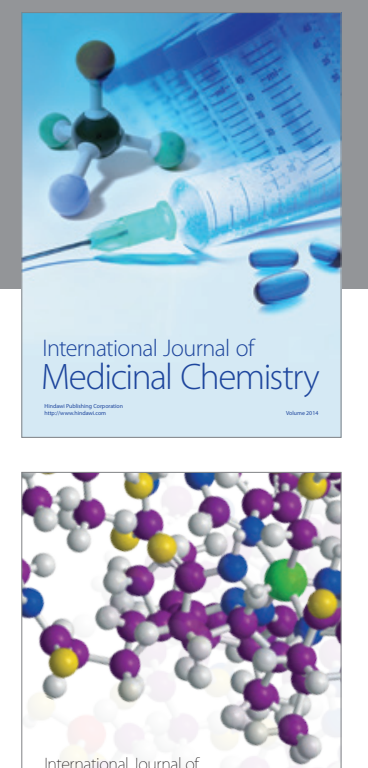

\section{Carbohydrate} Chemistry

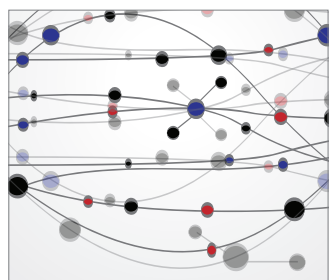

The Scientific World Journal
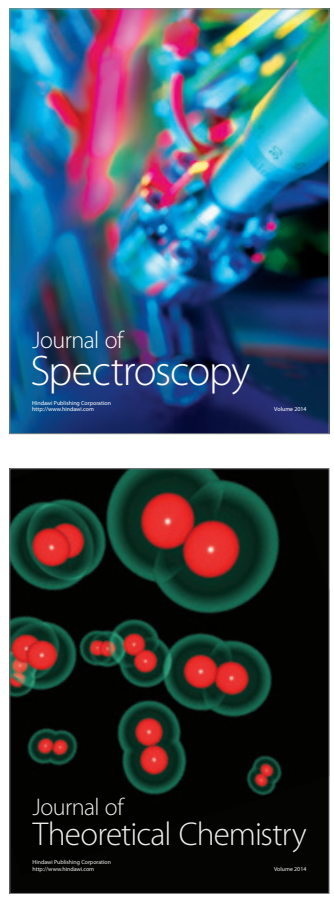
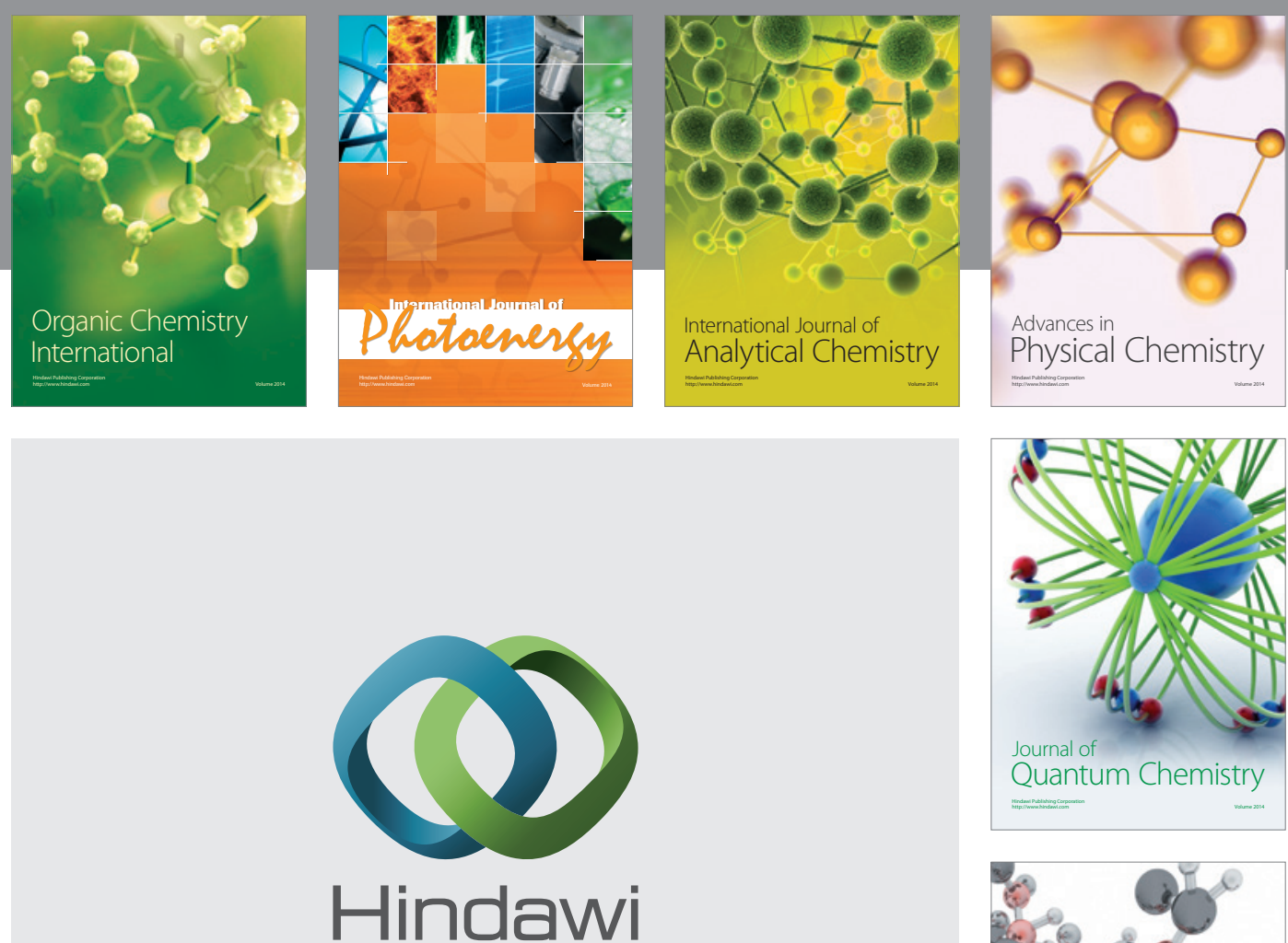

Submit your manuscripts at

http://www.hindawi.com

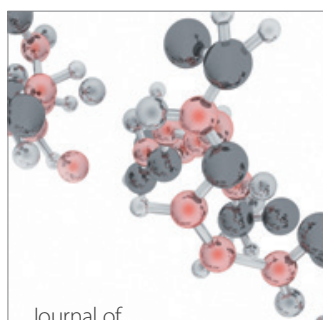

Analytical Methods

in Chemistry

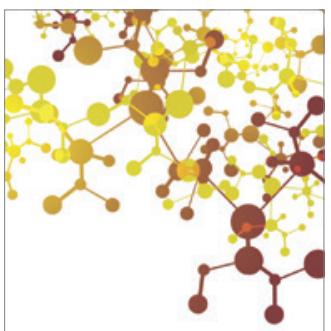

Journal of

Applied Chemistry

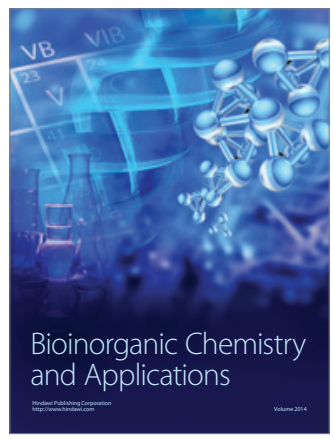

Inorganic Chemistry
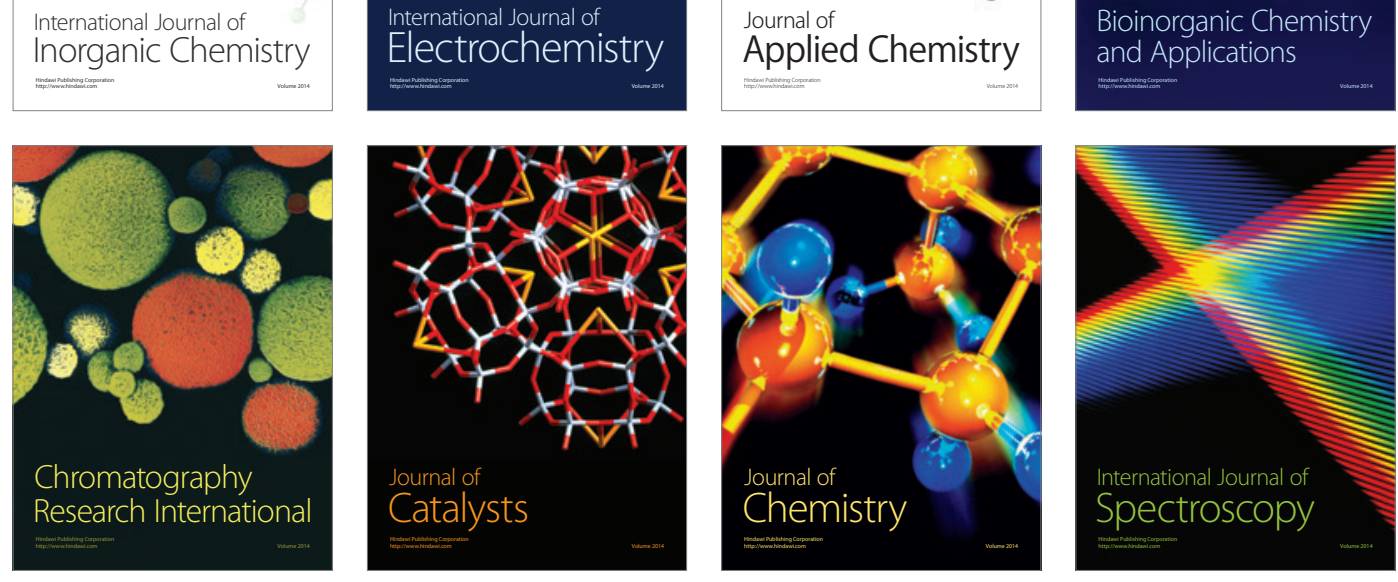